\title{
Design and Development of Intelligent Monitoring System for Plastic Tea Greenhouse
}

\author{
Fengyun Wang ${ }^{1}$, Jiye Zheng ${ }^{1}$, Lin $\mathrm{Mei}^{1}$, Zhaotang Ding ${ }^{2}$, Wenjie Feng ${ }^{1}$, \\ and Lei Wang, \\ ${ }^{1}$ S\&T Information Engineering Research Center, Shandong Academy of Agricultural Sciences, \\ Jinan 250100, Shandong Province, P.R. China \\ ${ }^{2}$ Tea Research Institute, Qingdao Agricultural University, Qingdao 266109, Shandong \\ Province, P.R. China \\ \{wfylily, jiyezheng, meilin670607, dzttea\}@163.com, \\ fengwjcn@qq.com, nkywl@126.com
}

\begin{abstract}
The article studies the growth environment conditions of tea and puts forward a wireless intelligent monitoring system for plastic tea greenhouse. According to the environmental requirements of tea tree, this article firstly defines the environmental parameters for planting tea in the greenhouse, and then designs the hardware and software of the intelligent monitoring system of tea in the greenhouse, and finally concludes the characteristics of this system. Through the practical application in tea greenhouse, the system decreases the cost and workload of tea planting and improves the quality of tea so as to enhance the economic returns.
\end{abstract}

Keywords: Tea, Wireless, Greenhouse, Monitoring.

\section{Introduction}

Camellia sinensis (L.) belongs to evergreen plant in subtropics and mostly grows among forestry community in the evergreen broad leaf rainforest and monsoon forest. It grows under the forest canopy for long time and has formed the ecological habit of shade-humid-tolerance and diffused light hobby [1].

In order to meet the requirements of famous high-quality tea, tea tree is planted in plastic greenhouse in the north area of the Yangtze River in our country, especially in Shandong and Henan etc. The plastic tea greenhouse is a facility cultivation form to use plastic film covering the tea garden. It is a new type of cultivation techniques to make the picking time of tea earlier in winner and spring when the temperature is lower or in the tea area where is subject to damage of cold spell in later spring. The technique can shorten the winter dormancy time to make the tea tree sprout earlier,

\footnotetext{
* Corresponding author. Supported by: National science and technology support plan of P.R. China (2011BAD21B06), Key Applied Technological Innovation Project in Agriculture of Shandong Province: Key Technology Study and Application of Regulation and Control between Fertilizer and Water of Tea with Good Quality and High Yield.
} 
add the picking turn of tea leaf and boost the shoot growth so as to improve the tea leaf yield. In addition, the technique controls the growth of tea tree through creating the suitable environmental conditions. Therefore it can be used to plant the tea in saline-alkali soil and desert etc non-cultivable land which can greatly improve the resource utilization rate of land, water and light [2] so as to realize the high yield, high quality, high efficiency and sustainable development of agriculture. The greenhouse cultivation of tea has become one of the basic modes for tea cultivation in north area of the Yangtze River [3].

In general, the quality of fresh tea leaf is directly decided by its ecological environment [4]. The change of environmental factors e.g. light intensity, air temperature and humidity and soil fertility etc will affect the growth and quality of tea leaf [5]. The plastic greenhouse belongs to the enclosed or semienclosed structure which can use the automation, computer, electronics etc technology to regulate and improve the temperature, humidity or light intensity etc parameters required by the growth of tea tree [6] to create the suitable growth conditions.

The wide use of wireless technology has made its application research in many national economy domains develop quickly since 1990s, especially the internet of things system based on zigbee wireless technology made it possible that the technology of precision agriculture was widely used for the production. In this article, a wireless intelligent monitoring system for plastic tea greenhouse based on zigbee is designed.

\section{Environmental Parameters and Control Requirements for Tea Tree}

\subsection{Temperature}

As a subtropical plant, the suitable temperature for tea tree is $17 \sim 25^{\circ} \mathrm{C}$. Too high or too low temperature can cause the yield reduction of tea leaf even no yield. The temperature should be controlled at about $25^{\circ} \mathrm{C}$ in daytime and not less than $8^{\circ} \mathrm{C}$ in nighttime. When the temperature within the greenhouse rises to $25^{\circ} \mathrm{C}$ in winter or $30^{\circ} \mathrm{C}$ in spring, it should be cooled by ventilation. When the temperature lowers under $20^{\circ} \mathrm{C}$, the ventilation port is closed to keep warm. In general, the ventilation port should be opened at about 10:00 am in fine day and closed at about 3:00 pm. When the temperature drops rapidly in nighttime, the straw mat should used to keep warm, if necessary, it is warmed by manual [7].

\subsection{Humidity}

The soil water content suitable to the growth of tea tree is $70 \sim 80 \%$. If it exceeds $90 \%$, the gas permeability of soil is bad which isn't beneficial to the growth of tea tree. The suitable air humidity within the greenhouse is $65 \sim 75 \%$ in daytime and about $80 \%$ in nighttime [8]. The ventilation opening lowers the air humidity and spray irrigation increases the air humidity. 


\subsection{Light Intensity}

The photosynthetic rate, maximum photosynthetic rate and apparent quantum yield of cutting seeding of tea tree are up to the maximum values at $75 \%$ natural light intensity. The chlorophyll content lowers with the increasing of light intensity. The chlorophyll $\mathrm{a} / \mathrm{b}$ increases with the increasing of light intensity. The new biomass is in line with the maximum photosynthetic which is up to the maximum value at $75 \%$ natural light intensity [9]. Because of sheltering of vertical shaft and arching within the greenhouse and weakening of light intensity caused by the reflecting, absorbing and refraction of transparent plastic etc, the light intensity within the greenhouse is only about $50 \%$ of natural light intensity which will affect the photosynthetic efficiency of tea leaf. Except for selecting apricus tea garden and using euphotic, anti-aging and antipollution transparent plastic film, artificial supplementary lighting is the most effective method that improves the light condition within the greenhouse in winter. The method is that use agricultural high pressure mercury lamp to illuminate the tea garden in the morning and evening in fine day or in cloudy day or in rainy day [10].

\subsection{Carbon Dioxide}

Carbon dioxide is the important matter for photosynthesis of tea tree. Carbon dioxide insufficient is the main limiting factor for yield and quality of tea leaf in plastic tea greenhouse. Before sunrise, the $\mathrm{CO}_{2}$ concentration within the greenhouse is up to $0.05 \% \sim 0.06 \%$ because of released $\mathrm{CO}_{2}$ by respiration of tea tree, microbial activities in the soil and organism decomposition (the $\mathrm{CO}_{2}$ concentration outside is ordinarily about $0.03 \%$ and stable). After sunrise, the $\mathrm{CO}_{2}$ concentration within the greenhouse drops remarkably with the enhancement of photosynthesis of tea tree. If it isn't ventilated in sunny day, the $\mathrm{CO}_{2}$ concentration even drops under $100 \mu \mathrm{g} / \mathrm{mL}$ and the tea tree almost can't carry our photosynthesis. Therefore $\mathrm{CO}_{2}$ fertilizer is applied within the greenhouse which can promote the photosynthesis of tea tree to improve the yield and quality of tea leaf. [11].

\section{$3 \quad$ Intelligent Monitoring System Design}

\subsection{System Structure}

In according to the environmental conditions and control requirements, the main environmental factors that affect the quality of tea are air temperature, air humidity, soil humidity, light intensity and $\mathrm{CO}_{2}$ concentration. Therefore there are mainly four basic parameters. If necessary, the outdoor meteorological station (mainly including outdoor temperature, humidity, wind direction, wind speed, rainfall and light intensity) and video monitoring may be set. The output devices include ventilation port, straw mat (or cotton quilt), high pressure mercury lamp and $\mathrm{CO}_{2}$ fertilizer distributor etc.

The system consists of wireless sensor networks (WSN), remote data transmission, monitoring center and wireless control output. The structure is shown in Fig. 1. 


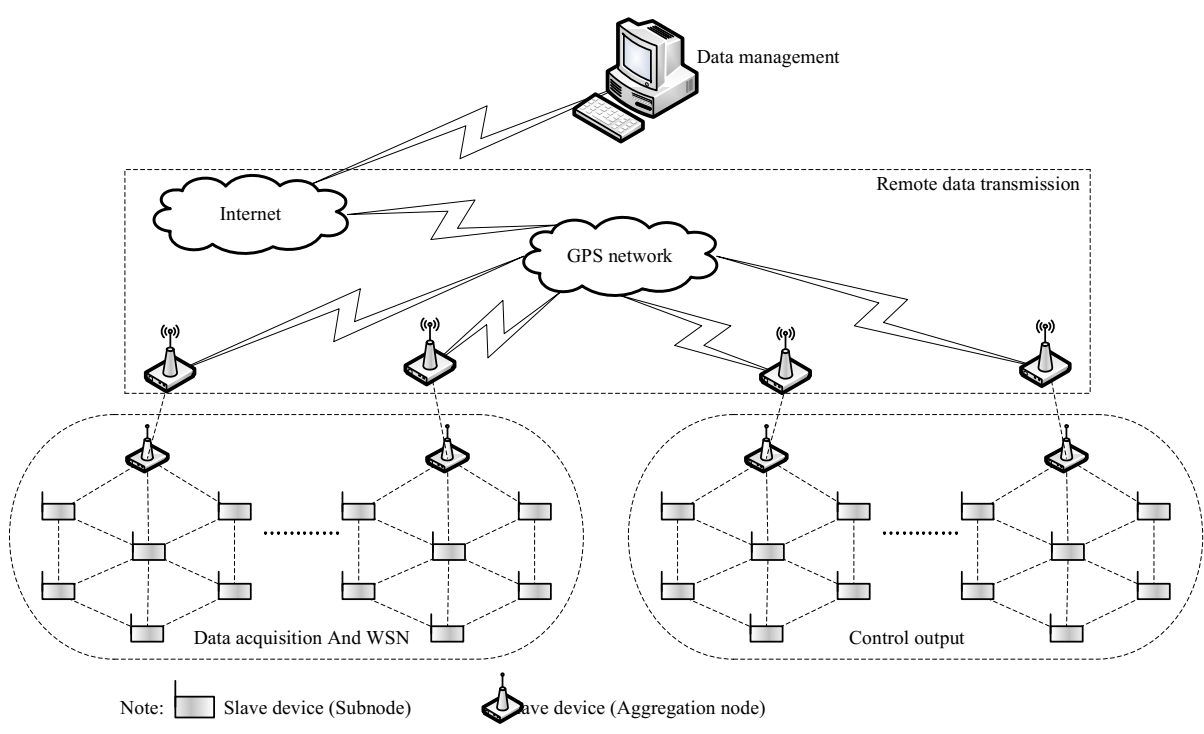

Fig. 1. Intelligent monitoring system structure

\subsection{Wireless Sensor Networks}

The wireless sensor networks mainly automatically acquire the air temperature, air humidity, soil humidity, light intensity, $\mathrm{CO}_{2}$ concentration and video information etc and send the data to total node by zigbee network rapidly and reliably.

It consists of sensor, subnode and aggregation node. The sensor perceives the environmental information and has high stability, strong capacity of resisting disturbance, high measurement accuracy and rapid response speed etc features.

The subnode and the aggregation node adopt zigbee technology. It is a duplex wireless communication technology with close distance, low complexity, low power consumption, low speed and low cost. The technology is mainly used for the transmission of periodical data, intermittent data and data with long response time. Zigbee technology has the following features:

$>$ Low power consumption. Two \#5 dry cells can support one node working for 6 26 months, even longer, which is its outstanding advantage.

$>$ Rapid response. It usually needs $15 \mathrm{~ms}$ only from sleep mode to working mode (Bluetooth needs $3-10$ s) and the node only needs $30 \mathrm{~ms}$ into the network which save the electric energy further.

$>$ Large network capacity. It can adopt star, tree and net three network structures. One main node manages a number of subnodes, up to 254 subnodes. At the same time one main node can be managed by upper node. Therefore it can compose of 65000 nodes at the most.

Because the system for plastic tea greenhouse needs a few sensors and close communication distance, the network adopts star topology structure which algorithm is simple and can meet the monitoring requirements of tea garden to a large extent. 
Considering the cost and performance, CC2530 is selected. It integrates the microprocessor module and a wireless transceiver module in one single chip. It has enhanced 8051 kernel with high speed, the latest zigbee 2007pro protocol, 2v-3.6v power supply range etc merits.

\subsection{Remote Data Transmission}

The data acquired from the sensors on site is sent to aggregation node by zigbee wireless communication technology. The aggregation node connects to the internet by GPRS network through which the data is sent to remote data monitoring center. At the same time, the order from monitoring center is sent to control devices by GPRS network so as to realize the remote intelligent monitoring for plastic tea greenhouse.

\subsection{Monitoring Center}

The monitoring center is the core of data management which realizes the analysis, processing, display, storage and output for acquired environmental data and releases it on the web. The monitoring software uses Microsoft visual basic 6.0 to develop and has the following functions:

$>$ Collect and record temperature, humidity, light intensity and $\mathrm{CO}_{2}$ concentration etc parameters, display them by digit, graph and image etc many modes and store them.

$>$ Device state display. The system displays the running state of control device, e.g. the open or close of ventilation port etc.

$>$ Set the alarm limit value of important parameters. When the value of alarmed parameter exceeds the limit, it will alarm automatically. The alarm mode includes multi-media sound-light alarm, network client alarm and mobile phone short message alarm etc.

$>$ Set the control modes. The control modes include manual and automatic two modes. The automatic mode includes time control and condition control. When the control device needs start or stop at fixed time, e.g. roll up the straw mat at 10:00 am in winter, the time control mode may be selected. When the device is controlled by temperature or humidity etc parameters e.g. open the ventilation port for air temperature over $30^{\circ} \mathrm{C}$, the condition control mode may be selected.

$>$ History data display. It includes the history information of parameters, device state, alarm and control modes etc. The user can make a period of history data into curve to reflect the changes in plastic tea greenhouse.

$>$ System configuration. It includes user management, communication set, parameter set, alarm value set, control conditions set, device set etc functions.

\subsection{Control Output}

The system adopts sectional-control strategy to decide the output. The sectional strategy uses different control algorithm in the deviation domain. Its block diagram is shown in Fig. 2. 


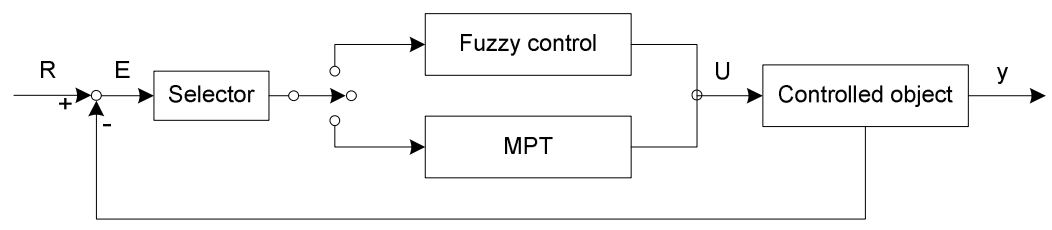

Fig. 2. Block diagram of sectional strategy

As shown in Fig. 2, when the deviation is larger than a certain domain, the fuzzy control is selected by operation mode selector to overcome integral saturation and confirm the output by fuzzy rules. When the deviation reduces within a certain domain, selector switches to MPT algorithm to decrease the overshooting during responding and eventually eliminate the residual. Therefore the sectional strategy integrates the merits of MPT algorithm and fuzzy control to improve the sensitivity and accuracy and obtain the ideal control effect [12].

The data is analyzed in according to the alarm set conditions and control algorithm. The result is sent to the control device or alarm device, e.g. ventilation port, straw mat, irrigating device and sound-light alarm device etc. For example, if the set value of air temperature is $25 \%$ within the greenhouse in winter for alarm and control, when the temperature rises to $25^{\circ} \mathrm{C}$, the system decides the output through analysis, then the ventilation port is opened by the driven motor and the system sends out alarm signal. The alarm signal is reset by confirmation and when the temperature falls to $25^{\circ} \mathrm{C}$, the ventilation port is closed.

\section{Conclusion}

The wireless intelligent monitoring system uses the latest wireless communication technology with micropower, digitalized temperature, humidity, light and $\mathrm{CO}_{2}$ sensing technology to monitor the key points in tea garden which greatly reduces the workload and cost and forecasts the unsafe state in advance. The system can promote the production and management level, improve the state of tea tree growth and reduce the diseases and insect pests so as to regulate the growth period and enhance the economic performance.

\section{References}

1. Gong, X.F., Yu, Y.B., Xiao, B., Chen, C., Jin, S.: Effects of Different Cultivating Modes of Tea Gardens on Environment and Tea Quality. Acta Botanica Boreali-Occidentalia Sinica 28(12), 2485-2491 (2008)

2. Feng, M.X., Wang, P.S., Jiang, R.D.: Description on tea insect pests and diseases in Laoshan tea area of Shandong Province. China Tea 24(2), 14-15 (2002)

3. Chen, J.R.: Overview of Tea Garden Production Technology in Plastic Greenhouse. Tea Science and Technology (1), 12-14 (2012) 
4. Shao, C.G., Yin, Z.X.: Research of Quality and Environmental Condition for Tea Leaf. Journal of Tea Business 21(4), 15-17 (1999)

5. Li, C.H., Wang, Y., Tang, X.B., Wang, Y.C.: The Effect of Different Eco-environment on the Quality of Famous Tea. Southwest China Journal of Agricultural Sciences 19(5), 946-948 (2006)

6. Wang, S.B.: Plastic Greenhouse Tea Garden's Management Technology. Hubei Agricultural Sciences 50(4), 754-758 (2011)

7. Guan, X.L., Zeng, L., Luo, L.Y.: Research Situation of Plastic Tea Greenhouse. Fujian Tea (4), 22-24 (2009)

8. Zheng, Y.Q.: Facility horticulture - Technical Points of Production Cultivation Management for Green Tea in North Area. Agriculture \& Technology 25(2), 145-146 (2005)

9. Gu, B.J., Chang, J., Zeng, J.M., Wang, L.Y., Yuan, H.B., Ge, Y., Liao, J.X., Zhou, J., Cheng, H.: Under Greenhouse Manufacturing Administration Studies on the Optimal Irradiation for Tea Seedlings. Journal of Tea Science 26(1), 24-30 (2006)

10. Han, W.Y., Yu, Y.W., Wang, G.Q.: Synthetical Cultivation Technology of Plastic Tea Greenhouse. China Tea (6), 4-5 (1997)

11. Jin, J., Luo, Y.P.: Research Progress for Photosynthesis of Tea. Tea Science and Technology (1), 1-5 (2002)

12. Wang, F.Y., Mei, L., Feng, W.J., Wang, L., Wang, L.M., Ruan, H.J.: A Greenhouse Control with Sectional-control Strategy Based on MPT Intelligent Algorithm. In: Li, D., Chen, Y. (eds.) CCTA 2012, Part I. IFIP AICT, vol. 392, pp. 43-50. Springer, Heidelberg (2013) 